\title{
Polaritons in Defect-Containing Lattice of Coupled Micro Resonators
}

\section{Rumyantsev V*}

Department of Dynamical Properties of Complex Systems, AA Galkin Donetsk Institute for Physics \& Engineering, Donetsk, 83114, Ukraine

\section{Editorial}

Results in crystal optics obtained during the past fifty years provide a solid foundation for the progress of modern photonics. Concepts developed in the physics of crystalline solids can potentially be applied to the physics of photonic super crystals. While the theory of impurity bands and excitons in semiconductor crystals has been constructed in 1970-1980, an analogous theory for photonic crystals is yet to be completed. Recent experiments and theoretical investigations reveal an intense interest for polartonic structures and systems of coupled micro resonators [1], whose applications include fabrication of clockworks of unprecedented accuracy [2,3] as well as the sources of coherent irradiation. There has been a significant advance in the photonics of imperfect structures. A number of our recent works have been devoted to optical activity of imperfect photonic crystals [4] and to dispersion of exciton-like electromagnetic excitations in non-ideal lattices of coupled micro resonators $[5,6]$.

Designing and utilization of novel materials for manufacturing of the sources of coherent irradiation is currently a vast interdisciplinary area, which spans various theoretical and fundamental aspects of laser physics, condensed matter physics, nanotechnology, chemistry as well information science $[7,8]$. Physical realization of corresponding devices requires the ability to manipulate the group velocity of propagation of electromagnetic pulses, which is accomplished by the use of the so-called polaritonic crystals. The latter represent a particular type of photonic crystals featured by a strong coupling between quantum excitations in a medium (excitons) and optical fields.

An example of polaritonic structure can be given by a spatially periodic system of coupled microcavities [9]. An interest for optical modes in microcavity arrays has been growing lately due to the enhancement of optoelectronic devices $[10,11]$. In this connection the defect-based resonators in photonic crystals deserve special attention. In [12] it was shown that such resonators can form a strong coupling with quantum dots. Alodjants [1] gave a theoretical analysis of the formation of quantum solitons coupled to lower dispersion branch (LDB) polaritons in a chain of microcavities. The authors suggest that such systems can be particularly appealing for the purposes of quantum information processing. Microcavity systems can also be employed for the construction of highly accurate optical clockworks [2,3].

It is worth stressing that the conventional polaritonic model $[1,5]$ of the atomic-optical interaction is only applicable to the case of ultracold atoms with frozen-out degrees of freedom. The corresponding approximation is valid when the number of atoms contained in individual cavities is relatively small $\left(\mathrm{N} \leq 10^{4}\right)$ [13]. Parameter $g$ of the strong atomic-optical interaction must satisfy the condition $g>>2 \pi / \tau_{\text {coh }}$

i.e. in each cavity $g$ should much exceed the inverse coherence time $\tau_{c o h}$ of the atomic-optical system. Physically, $\tau_{c o h}$ is the time of thermodynamic equilibration of the atomic system, which interacts with a quantized field in a polaritonic crystal? The said inequality holds at temperatures of the order of several $\mathrm{mK}$, when the spectral line broadening is negligible and so there are pure (thermodynamically equilibrium) quantum states of the atomic-field system.

The currently rapidly evolving field of endeavor is the photonics of imperfect structures. Some of our recent works are devoted to optical activity of imperfect photonic crystals [4] as well as to dispersion of exciton-like electromagnetic excitations in nonideal (defect-containing) arrays of coupled microcavities [5,6]. Introduction of defects provides an additional powerful tool for controlling the propagation of electromagnetic excitations through photonic structures.

In what follows we use the previously developed concepts of photonic structures $[5,6,14]$ to investigate a nonideal polaritonic crystal constituted by a topologically ordered assembly of coupled microcavities with embedded quantum dots. Quantum dots interact with resonator-localized quantized electromagnetic fields and each of tunnel-coupled resonators possesses a single optical mode. It is assumed that certain portions of quantum dots and cavities are missing, which is viewed as the presence of defects. The polariton spectrum of the structure and the related quantities of interest (the band gap, the effective masses of polaritons as well as their densities of states) are investigated as functions of defect concentrations.

We consider a non-ideal polaritonic crystal that is a set of spatially ordered microcavities containing atomic clusters or quantum dots. Numerical modeling of the dispersion of polaritons in this imperfect lattice for different defect concentrations has been performed using the virtual crystal approximation $[15,16]$. Here we assume that the spatial distribution of cavities is translation invariant, while the atomic subsystem has randomly distributed defects: impurity atomic clusters (quantum dots) or vacancies (Figure 1).

Analytical expressions for polariton eigen-frequencies, effective masses and group velocities as functions of the quantum dot and vacancies concentrations have been obtained. It turns out that even with a small number of vacancies in the lattice (one vacancy per one thousand cavities) the polariton effective mass may be increased by three orders of magnitude [17].

In Figure 2a the dispersion bands $\Omega_{ \pm}\left(k_{x}, k_{y}, C_{1}{ }^{\mathrm{v}}, C_{2}{ }^{\mathrm{v}}\right)$ are plotted for an ideal structure $\left(C_{1} \mathrm{v}, C_{2}^{\mathrm{v}}\right.$ denote defect concentrations of the atomic or resonator subsystems correspondently), Figure $2 \mathrm{~b}$ illustrates their transformation under the decrease of parameter $g$ (responsible for interaction between the atomic and photonic subsystems) by a factor of 10. Figure $2 c$ gives an example of dispersion bands of a nonideal structure $\left(C_{1} \mathrm{v}=0.43, C_{2}^{\mathrm{v}}=0.2\right)$.

Our model is primarily based on the virtual crystal approximation, which is often employed to examine quasiparticle excitations in

*Corresponding author: Rumyantsev V, Department of dynamical properties of complex systems, AA Galkin Donetsk Institute for Physics and Engineering, Donetsk, 83114, Ukraine, Tel: +380-62311-5277; E-mail: 380957931135@yandex.ru

Received May 10, 2016; Accepted May 13, 2016; Published May 16, 2016

Citation: Rumyantsev V (2016) Polaritons in Defect-Containing Lattice of Coupled Micro Resonators. J Laser Opt Photonics 3: e108. doi:10.4172/2469410X.1000e108

Copyright: (C) 2016 Rumyantsev V. This is an open-access article distributed under the terms of the Creative Commons Attribution License, which permits unrestricted use, distribution, and reproduction in any medium, provided the original author and source are credited. 


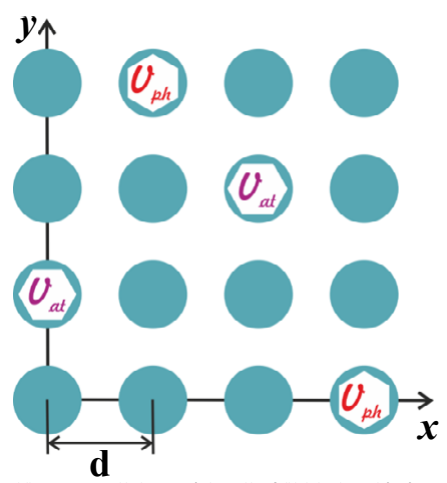

Figure 1: Schematic of a modeling square one-sublattice array of microcavities. Solid circles denote defect-free cavities with embedded quantum dots. $V_{a t}$-type of defect is a cavity with a missing quantum dot. $V_{p}$-type of defect is an empty site with no cavity (and hence with no quantum dot, since atomic clusters can only reside at the existent cavities). Defect concentration in the atomic subsystem (i.e. concentration of sites with missing quantum dots) $C_{1}{ }^{V}$ equals to the sum of concentrations of the $V_{a t}$ and $V_{p h}$-types of defects. Defect concentration in the photonic subsystem (concentration of sites with missing cavities) $C_{2}{ }^{v}$ equals to the $V_{p h}$-type defect concentration. There holds an obvious inequality $C_{1}{ }^{v} \geqslant C_{2}{ }^{v}$.

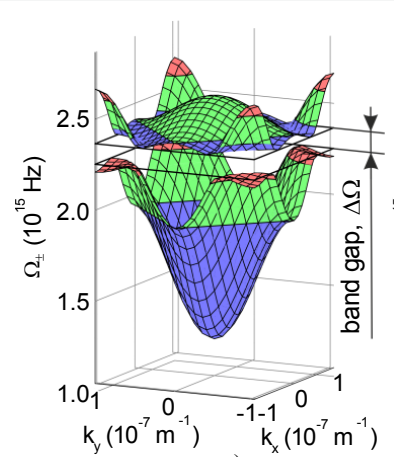

a)

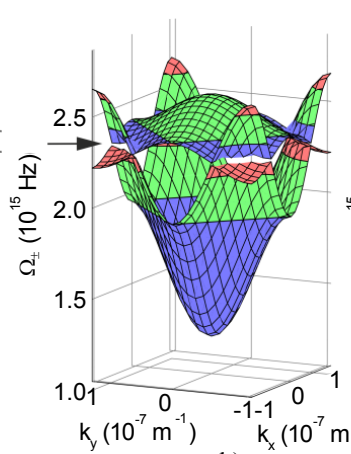

b)

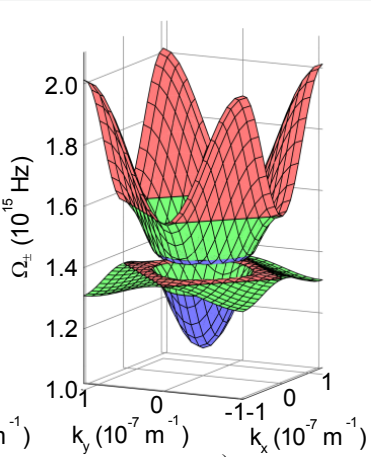

Figure 2: Polariton energy bands of an ideal $(\mathrm{a}, \mathrm{b})$ microcavity array $\left.C_{1}{ }^{\mathrm{v}}=C_{2}{ }^{\mathrm{v}}=0\right)$ and a defect-containing $(\mathrm{c})$ array $\left(C_{1}{ }^{\mathrm{v}}=0.43, C_{2}{ }^{\mathrm{V}}=0.2\right)$. (b) Illustrates the narrowed "bottle neck" resulting from the decrease of the parameter $g$ responsible for interaction between the atomic and photonic subsystems by a factor of 10 .

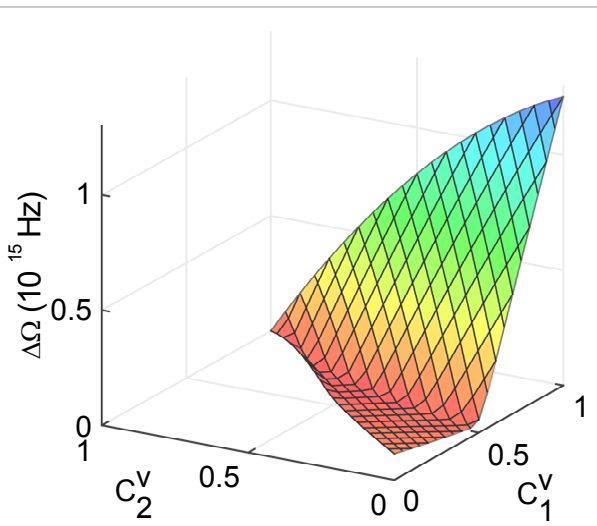

Figure 3: Band gap width plotted as a function of defect concentrations in the domain of definition $C_{1}{ }^{v} \geqslant C_{2}$.

sufficiently simple disordered superstructures. More complex systems usually require the use of more sophisticated methods such as the (one- or multinode) coherent potential approximation [16], the averaged T-matrix method and their various modifications. The obtained numerical results contribute to our understanding of composite polaritonic structures and the prospects of their utilization for construction of solid-state devices with controllable propagation of electromagnetic waves (Figure 3).

\section{References}

1. Alodjants AP, Barinov IO, Arakelian SM (2010) Strongly localized polaritons in an array of trapped two-level atoms interacting with a light field. J Phys B: A Mol Opt Phys 43: 1-16.

2. Hou D, Ning B, Wu J, Wang Z, Zhao J (2013) Demonstration of a stable erbiumfiber-laser-based frequency comb based on a single rubidium atomic resonator. Appl Phys Lett 102.

3. Papp SB, Beha K, Del'Haye P, Quinlan F, Lee H, et al. (2014) Microresonator frequency comb optical clock. Optica 1: 10-14.

4. Rumyantsev VV, Fedorov SA, Gumennyk KV, Proskurenko MV (2014) Peculiarities of propagation of electromagnetic excitation through a nonideal gyrotropic photonic crystal. Physica B: Condensed Matter 442: 57-59.

5. Rumyantsev VV, Fedorov SA, Gumennyk KV, Sychanova MV, Kavokin AV (2014) Exciton-like electromagnetic excitations in non-ideal microcavity supercrystals. Nature Sci Rep 4.

6. Rumyantsev VV, Fedorov SA, Gumennyk KV, Sychanova MV (2015) Dispersion characteristics of electromagnetic excitations in a disordered $1 \mathrm{D}$ lattice of coupled microresonators. Physica B 461: 32-37.

7. Cai W, Shalaev V (2010) Optical Metamaterials: Fundamentals and Applications, Springer-Verlag, New York, USA.

8. Razeghi M (2009) Technology of Quantum Devices, Springer, New York, USA

9. Vahala KJ (2003) Optical microcavities. Nature 424: 839.

10. Kaliteevskii MA (1997) Coupled vertical microcavities. Tech Phys Lett 23: 120

11. Golubev VG, Dukin AA, Medvedev AV, Pevtsov AB, Sel'kin AV et al. 
Citation: Rumyantsev V (2016) Polaritons in Defect-Containing Lattice of Coupled Micro Resonators. J Laser Opt Photonics 3: e108. doi:10.4172/2469$410 \mathrm{X} .1000 \mathrm{e} 108$

Page 3 of 3

(2003) Splitting of resonant optical modes in Fabry-Perot microcavities. Semiconductors 37 : 832-837.

12. Englund D, Majumdar A, Faraon A, Toishi M, Stoltz N, et al. (2010) Resonant Excitation of a Quantum Dot Strongly Coupled to a Photonic Crystal Nanocavity. Phys Rev Lett 104

13. Anglin JR, Vardi A (2001) Dynamics of a two-mode Bose-Einstein condensate beyond mean-field theory. Phys Rev A 64.

14. Rumyantsev VV, Fedorov SA, Gumennyk KV, Sychanova MV, Kavokin AV
(2016) Polaritons in a nonideal periodic array of microcavities. Superlattices and Microstructures 89: 409-418.

15. Ziman JM (1979) Models of disorder: The theoretical physics of homogeneously disordered systems, Cambridge University Press, Cambridge.

16. Los VF (1987) Projection operator method in the theory of disordered systems I Spectra of quasiparticles Theor Math Phys 73: 1076-1088.

17. Alodjants AP, Rumyantsev VV, Fedorov SA, Proskurenko MV (2014) Polariton Dispersion Dependence on Concentration of Admixture in Imperfect Superlattice of Coupled Microresonators. Functional Materials 21: 211-216. 